4 Manuel Pascal Martin ${ }^{1}$, Stéphane Cordier ${ }^{2}$, Jérôme Balesdent ${ }^{3}$ and Dominique Arrouays ${ }^{1}$.

$5 \quad$ INRA, Unité Infosol, 2163 Ave Pomme de Pin, BP 20619, F-45166 Olivet, France

\section{Periodic solutions for soil carbon dynamics equilibriums with time-varying forcing variables}

${ }^{2}$ MAPMO UMR CNRS 6628 Université d’Orléans 45067 Orléans France.

${ }^{3}$ LEMIR, UMR 6191 CEA Cadarache 13108 St Paul lez Durance France.

\section{Abstract}

Numerical models that simulate the dynamics of carbon in soil are increasingly used to improve our knowledge and help our management of the carbon cycle. Calculation of the long term behavior of these models is necessary in many applications but encounters the difficulty of managing the periodic forcing variables, e.g., seasonal variations, such as carbon inputs and decomposition rates. This calculation is conventionally done by running the model over large time durations or by assuming constant forcing variables. Two methods, which make it possible to rapidly compute periodic solutions taking into account the time variations of these variables, are proposed. The first one works on discrete-time models and the second one on continuous-time models involving Fourier transforms. Both methods were tested on the Rothamsted carbon model (RothC), a discrete-time model which has also been given a continuous approximation, using realistic an unrealistic sets of time-varying forcing functions. Both methods provided an efficient way to compute the periodic solutions of the RothC model within the application domain of the model. Compared to running the discrete model to the equilibrium, reduction in the computational cost was of up to $95 \%$ at the expense of a maximum absolute error of $1 \%$ for the estimation of carbon stocks. For specific distributions of the forcing variables the use of Fourier transform of zero order, which was equivalent to assume constant forcing variables, led to a maximum absolute error of $55 \%$ in the estimation of the long term behavior of the model. There, a Fourier transform of order higher than zero is required.

Keywords: soil organic carbon dynamics, discrete formulation, continuous formulation, steady state, periodic solutions, linear model, Fourier series. 


\section{Introduction}

\section{1. carbon dynamics}

The soil organic carbon (SOC) plays an important role in several environmental and land management issues. One of the most important issues is the role that SOC plays as part of the terrestrial carbon and might play as a regulator of the atmospheric $\mathrm{CO} 2$. Many factors are likely, in a near future, to modify the SOC content, including changes in agricultural practices (Betts, 2000; Vleeshouwers and Verhagen, 2002; Bellamy et al., 2005) and global climate changes (Jenkinson et al., 1991; Cao and Woodward, 1998; Cox et al., 2000; Jones et al., 2005; Knorr et al., 2005). Understanding SOC and soil organic matter (SOM) dynamics as a function of soil characteristics, agricultural management and climatic conditions is therefore crucial, and many models have been developed in this perspective. Most models of SOM turnover, excepting a few (Bosatta and Agren, 2003), are compartmental models, exhibiting various degree of complexity. The compartments represent carbon originating from plants or contained in soil and transformed by microorganisms and each one is characterized by a particular decomposition rate representing more labile or more stable forms of soil organic matter. Some models include $\mathrm{N}$ turnover and/or plant growth modules (CENTURY) when others only focus on SOC (RothC). Also, most use a linear method of transferring quantities between the different compartments (Baisden and Amundson, 2003) but some models including non-linear dynamics have also been developed more recently (Manzoni et al., 2004).

These models are used in a variety of ways and often for long term studies (Coleman et al., 1997; Falloon and Smith, 2002; Franko et al., 2002; Shevtsova et al., 2003; Shirato, 2005; Shirato et al., 2005b; Shirato and Yokozawa, 2005a). The behavior of the SOC system, over a long term and assuming that the environment of the system (inputs of organic carbon, climatic conditions) is stable, is reported to tend toward a steady state. Although many soils under study might not have reached equilibrium, being able to compute and predict the long-term solution is extremely valuable. It gives a synthetic view of the system in given agro-climatic conditions, makes it possible to test if a studied soil has reached an equilibrium or not, to envision what would be the consequences of specific events onto a given soil assuming that a new stable state is reached and to serve as a control case or initial conditions (Thornton and Rosenbloom, 2005). Technically, the equilibrium assumption is also commonly used to solve analytically mathematical systems. Such an analytical solution gives an explicit relationship between model inputs and outputs and may, in turn, be computed without simulating or 
integrating numerically the system until it reaches a stable state, thus saving computation time. When models cannot be formulated analytically, estimating the steady state solutions still can be useful and generic or model specific efficient numerical methods are available (Thornton et al., 2005). More generally, being able to use analytical forms of the long-term solutions is particularly useful in understanding models behavior and relationships between input and output variables of the model.

Some of the SOC models have been formulated mathematically (Parshotam, 1996; Bolker et al., 1998; Yang et al., 2002; Baisden et al., 2003; Manzoni et al., 2004) and approaches, as the development of the ICBM family models (Katterer and Andren, 2001), specifically aim at proposing analytically solved models representing the conventional wisdom of soil $\mathrm{C}$ and $\mathrm{N}$ modelling. For these models, when studying $\mathrm{N}$ and $\mathrm{C}$ soil content at steady state, it is usually assumed that forcing variables (typically climatic variables and variables representing inputs) can be set to their average value, calculated over a representative year for instance, which considerably eases the mathematical treatment. There, the long-term behavior of the model truly is a steady state. Consequences of such an assumption have for now been tested only empirically for some models and specific conditions. In some cases, environmental shifts from one stable state to another or brief events are considered and mathematical treatment used to estimate the new stable state after perturbation or the system resilience. We propose here two methods which make it possible to deal with continuously time varying agroclimatic conditions (e.g. forcing variables), when they can be specified as periodic functions. The first one works on discrete-time models and the second one on continuous-time models involving Fourier transforms. We considered the Rothamsted model with crop cultivations, as representative of many models of soil organic matter dynamics, anticipating that the second method could also be applied to models involving non-linear dynamics. We used these methods to test the consequences of assuming yearly constant agro climatic condition instead of considering their intra-annual variability.

\section{Methods}

\subsection{Discrete formulation}

The RothC model (Coleman et al., 1997) splits the soil carbon into four active compartments and one inactive. At each time step, the four active compartments, decomposable plant material, DPM, resistant plant material, RPM, the microbial community BIO and the humus, HUM, undergo decomposition as a function of a rate constant, depending on the compartment and on a rate modifier. The rate modifier depends on the clay content of the soil, climatic 
96 variables and land cover. Products of the decomposition are $\mathrm{CO}_{2}$ and carbon feeding the $\mathrm{BIO}$ 97 and HUM compartments. The fraction of the decomposed carbon incorporated into BIO and 98 HUM increases as a function of the clay content of the soil. Carbon enters the soil through the 99 DPM and RPM compartments. The fraction input in DPM and RPM respectively is chosen as 100 a constant which is an estimate of the decomposability of the plant material. It depends on the 101 cultivation being considered. The model can be formulated as

$$
C_{t+1}=F_{t} C_{t}+B_{t}
$$

102 Where

$$
\begin{aligned}
& C_{t}=\left(\begin{array}{c}
\text { dpm } \\
\text { rpm } \\
\text { bio } \\
\text { hum }
\end{array}\right)_{t} \\
& F_{t}=\left(\begin{array}{cccc}
e^{-\rho_{t} k_{d p m}} & 0 & 0 & 0 \\
0 & e^{-\rho_{t} k_{r p m}} & 0 & 0 \\
\alpha\left(1-e^{-\rho_{t} k_{d p m}}\right) & \alpha\left(1-e^{-\rho_{t} k_{\text {rpm }}}\right) & \alpha\left(1-e^{-\rho_{t} k_{\text {bio }}}\right)+e^{-\rho_{t} k_{b i o}} & \alpha\left(1-e^{-\rho_{t} k_{\text {hum }}}\right) \\
\beta\left(1-e^{-\rho_{t} k_{d p m}}\right) & \beta\left(1-e^{-\rho_{t} k_{r p m}}\right) & \beta\left(1-e^{-\rho_{t} k_{\text {bio }}}\right) & \beta\left(1-e^{-\rho_{t} k_{\text {hum }}}\right)+e^{-\rho_{t} k_{\text {hum }}}
\end{array}\right)
\end{aligned}
$$

103 and

$$
B_{t}=\left(\begin{array}{c}
a_{d p m} \\
a_{r p m} \\
a_{b i o} \\
a_{\text {hum }}
\end{array}\right) b_{t}
$$

104 The four input coefficients $\left(\mathrm{a}_{\mathrm{dpm}}, \mathrm{a}_{\mathrm{rpm}}, \mathrm{a}_{\mathrm{bio}}\right.$ and $\left.\mathrm{a}_{\mathrm{hum}}\right)$ sum up to 1 and in the most common case 105 one uses $a_{\mathrm{dpm}}=\gamma, \mathrm{a}_{\mathrm{rpm}}=1-\gamma, \mathrm{a}_{\mathrm{bio}}=0$ and $\mathrm{a}_{\mathrm{hum}}=0, \gamma$ depending on the quality of the plant 106 material.

107 Here, $\alpha$ and $\beta$ are fractions of metabolized C incorporated respectively into BIO and HUM. $b_{t}$ 108 is the carbon amount $\left(\mathrm{t}^{-h a^{-1}}\right)$ entering the system at month $\mathrm{t}, k_{i}$ the decomposition rate for 109 compartment $i$ and $\rho_{t}$ the rate modifier. 
111 In case where $b_{t}$ and $\rho_{t}$ are constant, one can demonstrate that the $\left(\mathrm{I}_{4}-\mathrm{F}\right)$ matrix, where the

112 matrix $\mathrm{I}_{4}$ is the 4-by-4 identity matrix, has an inverse (see below) and that the system yields a

113 steady state solution. Assuming that $F$ and $B$ are respectively the time constant carbon flows

114 and carbon inputs, one can write

$$
C^{*}=\left(I_{4}-F\right)^{-1} B
$$

115 However, usually $F_{t}$ and $B_{t}$ vary through time but it can be assumed that they have a periodic

116 behavior. Typically, if the agronomical practices are cyclic and if the weather conditions can

117 be considered as periodic, $\rho_{t}, b_{t}$, and consequently $F_{t}, B_{t}$ will also behave periodically.

118 Assuming that the periodicity of these variables is $P$, one looks for a solution of $\mathrm{C}$ such that

$$
C_{t+P}=C_{t}
$$

119 For example, considering the common case the case where $P$ is 12 months, we can write 120 down

$$
\begin{gathered}
C_{t+1}=F_{1} C_{t}+B_{1} \\
\cdot \\
C_{t+11}=F_{11} C_{t+10}+B_{11} \\
C_{t}=F_{12} C_{t+11}+B_{12}
\end{gathered}
$$

121 which can be reformulated as:

$$
\left(\begin{array}{ccccc}
0 & I_{4} & 0 & \cdots & 0 \\
\vdots & \ddots & \ddots & \ddots & \vdots \\
\vdots & & \ddots & \ddots & 0 \\
0 & \cdots & \cdots & 0 & I_{4} \\
I_{4} & 0 & \cdots & 0 & 0
\end{array}\right)\left(\begin{array}{c}
C_{t} \\
\vdots \\
\vdots \\
C_{t+10} \\
C_{t+11}
\end{array}\right)=\left(\begin{array}{ccccc}
F_{1} & 0 & \cdots & \cdots & 0 \\
0 & F_{2} & \ddots & & \cdots \\
\vdots & \ddots & \ddots & \ddots & \vdots \\
\vdots & & \ddots & \ddots & 0 \\
0 & \cdots & \cdots & 0 & F_{12}
\end{array}\right)\left(\begin{array}{c}
C_{t} \\
\vdots \\
\vdots \\
C_{t+10} \\
C_{t+11}
\end{array}\right)+\left(\begin{array}{c}
B_{1} \\
\vdots \\
\vdots \\
B_{11} \\
B_{12}
\end{array}\right)
$$

122 and finally yields:

$$
\left(\begin{array}{c}
C_{t} \\
\vdots \\
\vdots \\
\vdots \\
C_{t+11}
\end{array}\right)=-\left(\begin{array}{ccccc}
F_{1} & -I_{4} & 0 & \cdots & 0 \\
0 & F_{2} & -I_{4} & & \cdots \\
\vdots & \ddots & \ddots & \ddots & 0 \\
0 & & \ddots & \ddots & -I_{4} \\
-I_{4} & 0 & \cdots & 0 & F_{12}
\end{array}\right)^{-1}\left(\begin{array}{c}
B_{1} \\
\vdots \\
\vdots \\
\vdots \\
B_{12}
\end{array}\right)
$$

123 Solving this system (which can be performed via matrix inversion by common statistical 124 packages or spreadsheet programs) yields a vector of dimension $4 * 12$, which is the sequence 
125 of states $C_{i}=\left(\mathrm{dpm}_{i}, \mathrm{rpm}_{i}, \text { bio }_{i}, \text { hum }_{i}\right)^{T}, i$ in $|[1,12]|$ and which characterizes the oscillatory state

126 of carbon stock in each compartment, keeping track of the temporal variability of the forcing variables over the period.

128

129

130

131

132 with

$$
\begin{gathered}
A=\left(\begin{array}{cccc}
-k_{d p m} & 0 & 0 & 0 \\
0 & -k_{r p m} & 0 & 0 \\
\alpha k_{d p m} & \alpha k_{r p m} & (\alpha-1) k_{b i o} & \alpha k_{h u m} \\
\beta k_{d p m} & \beta k_{r p m} & \beta k_{b i o} & (\beta-1) k_{\text {hum }}
\end{array}\right) \\
B(t)=\left(\begin{array}{llll}
a_{d p m} & a_{r p m} & a_{b i o} & \left.a_{\text {hum }}\right)^{T} b(t)
\end{array}\right.
\end{gathered}
$$

$133 \rho(t)$ is the decomposition rate modifier. In the current RothC formulation, $\rho(t)$ is a function of 134 monthly rainfall, temperature, pan open evaporation and land cover, as well as percentage of 135 clay in the considered soil. When the time varying input variables (climatic and agricultural 136 variables) are considered as periodic functions of period $P, \rho(t)$ itself is a periodic function 137 with the same period. $b(t)$ is considered on a periodical basis too, as for the discrete 138 formulation of the model. Again, the system defined by $C(t)$ is expected to tend toward a 139 oscillatory state as $t \rightarrow+\infty$. Study of the eigenvalues of $\rho(t) A$ enables to characterize such a 140 behavior. Let us define $y(t)$ and $A(t)$.

$$
y(t)=e^{-A(t)} C(t)
$$

141 where $A(t)$ is the primitive of $\rho(t) A$.

142 From Eq.(4) and (6) we obtain

$$
y^{\prime}(t)=e^{-A(t)} B(t)
$$

143 and

$$
y(t)=y_{0}+\int_{0}^{t} e^{-A(s)} B(s) d s
$$

144 Setting $A(0)$ to the four by four zero matrix, from Eq.(6) and (7) it can be written 


$$
C(t)=e^{A(t)} C_{0}+e^{A(t)} \int_{0}^{t} e^{-A(s)} B(s) d s
$$

145 We can first observe that if $A(t)$ eigenvalues are negative, $C(t)$ as $t \rightarrow+\infty$ do not depend on 146 initial conditions $C_{0}$. Secondly, if $C(t)$ has a periodic solution, say $C_{0}$, with period $P$, it can be

147 shown using semigroup representations that it satisfies the following Equation,

$$
C_{0}=e^{A(T)} C_{0}+e^{A(T)} \int_{0}^{T} e^{-A(s)} B(s) d s
$$

148 A solution to this equation exists and is unique if $\left(I d-e^{-A(t)}\right)$ has an inverse, which can be

149 demonstrated to be true if $A$ is invertible. A sufficient condition for $A$ to be invertible is that

150 all its eigenvalues are nonpositive, i.e. using the definition of $A$ (Eq.(5)) when $\alpha+\beta<1$. This

151 condition is always true for the RothC model due to the definition of $\alpha$ and $\beta$ (Coleman and

152 Jenkinson, 1995) and thus the stock of carbon in each compartment tends towards a periodic

153 solution in large times whatever the input values are.

154 Characterization of the long-term behavior

155 Approximations of this behavior can be made using Fourier series. Setting

$$
C_{N}(t)=\sum_{k=-N}^{N} C_{k} e^{i k t}, \rho_{N}(t)=\sum_{k=-N}^{N} \rho_{k} e^{i k t} \text { and } b_{N}(t)=\sum_{k=-N}^{N} b_{k} e^{i k t}
$$

156 with

$$
\rho_{k}=\int_{0}^{2 \pi} \rho(s) e^{-i k s} d s \text { and } b_{k}=\int_{0}^{2 \pi} b(s) e^{-i k s} d s
$$

$157 \rho_{k}$ and $b_{k}$ coefficients can be obtained from the monthly input values used in the RothC model.

$158 C(t), A(t)$ and $B(t)$ can be replaced by their respective Fourier transform in Eq.(4) giving the

159 following approximation.

$$
\sum_{k=-N}^{N} i k C_{k} e^{i k t}=A \sum_{j, k / j+k=-N}^{N} C_{j} \rho_{k} e^{i(j+k) t}+\sum_{k=-N}^{N} b_{k} e^{i k t}
$$

160 Setting $N$, the order of the Fourier series, to zero, we calculate the $\mathrm{C}_{0}$ term.

$$
C_{0}=-A^{-1} B_{0} / \rho_{0}
$$

161 Assuming that $B_{0}=(\gamma, 1-\gamma, 0,0)^{T} . b_{0}$, which means that carbon inputs only to the DPM and 162 RPM compartments, leads to the following solution: 


$$
C_{0}=\left(\begin{array}{c}
\gamma b_{0} / \rho_{0} k_{d p m} \\
(1-\gamma) b_{0} / \rho_{0} k_{r p m} \\
b_{0} \alpha /\left[(1-\alpha-\beta) \rho_{0} k_{b i o}\right] \\
b_{0} \beta /\left[(1-\alpha-\beta) \rho_{0} k_{\text {hum }}\right]
\end{array}\right)
$$

163

164

165

166

167

168

169

170

171

172

173

174

175

176

177

$b_{0}$ and $\rho_{0}$ terms represent averages of $b(t)$ and $\rho(t)$ over the considered period, which is one year. This solution gives an explicit formulation of the long-term behavior of the system, which obviously equals what would have been found using the assumption of constant forcing variables set to the average values. This solution does not enable to takes into account the temporal variability of the carbon stocks throughout the year. This could have been achieved by computing the $\mathrm{C}_{1}$ term which itself is an approximation of the primary oscillations of the system's long-term behavior. Such a calculation lies beyond the scope of this paper. These oscillations directly depend on the temporal variability of the forcing variables but their size is usually small compared to the total SOC. In the following, they will be handled only with the discrete formulation of the RothC model (Eq.(3)).

\subsection{Comparison of the approaches}

Parshotam (1996) showed that given some restrictions the continuous formulation (Eq.(4)) is a good approximation of what would be the continuous formulation of the original discrete time RothC model. It is possible to turn this the other way round and say that the RothC model is an approximation of the discretization of the continuous model given in Eq.(4). Discretization of Eq.(4) leads to, in case of constant inputs during the sampling intervals (Parshotam, 1996):

$$
C_{(k+1) \Delta t}=e^{\rho(k \Delta t) A \Delta t} C_{k \Delta t}+(\rho(k \Delta t) A)^{-1}\left(e^{\rho(k \Delta t) A \Delta t}-I_{4}\right) B(k \Delta t)
$$

RothC is an approximation of the above equation because

$$
F_{t} \approx e^{\rho(k \Delta t) A \Delta t} \text { and } B_{t} \approx(\rho(k \Delta t) A)^{-1}\left(e^{\rho(k \Delta t) A \Delta t}-I_{4}\right) B(k \Delta t)
$$

Thus, the parameters used in the continuous model (e.g. $\alpha, \beta, k_{d p m}, k_{r p m}, k_{b i o}$ and $k_{h u m}$ ) should not be equated with those of the RothC model. However, numerically it makes little difference, and in the following developments, we shall do it.

Both approaches (using the discrete or the continuous formulation) can be used to characterize the periodic long-term behavior of the system. The first approach (Eq.(3)) uses the discrete formulation of the model which formally reproduces the specification of the RothC model contrary to the second one (Eq.(9)) which uses a continuous formulation and a zero order Fourier transform. This latest approach gives a simpler explicit solution, function 
of the input variables and parameters of the model $\left(\alpha, \beta, k_{d p m}, k_{r p m}, k_{b i o}\right.$ and $\left.k_{h u m}\right)$. It might also

190 be more interesting to work with the continuous form of the model because of its greater generality and since it is usually easier to discretize a continuous model rather than doing the opposite.

193 To assess the validity of both approaches in characterizing the long-term behavior with time194 varying forcing variables, we first compared both approaches between themselves and with the discrete model where variability of forcing is leveraged over the year. This was performed using a weather dataset composed by monthly averages calculated over 12 years (1992-2004) on a $0.125^{\circ}$ grid (4144 cells) covering the French country. For each point of the grid, we considered a unique crop system, with inputs being 0.50, 0.20, 0.10, 0.10, 0.10, $1.44 \mathrm{tC}^{-\mathrm{ha}^{-1}}$ respectively for each month from March to August, otherwise null with a bare soil (adapted from Swinnen et al., 1995 and Bolinder et al., 1997 for winter wheat). \%Clay was set to $10 \%$. The computation of the long-term behavior using the discrete formulation resulted in a periodic solution, i.e. a sequence of twelve $C_{i}$ states, $i$ in [1..12] (Eq.(3)). The solution given by a discrete formulation with averaged forcing variables was a single state noted $\mathrm{C}_{\mathrm{avg}}$, the calculation obtained using the continuous formulation was also a single state, noted $\mathrm{C}_{0}$ (Eq.(9)). We compared $\mathrm{C}_{\text {avg }}$, with $\mathrm{C}_{0}$ and with the average state of the $\mathrm{C}_{\mathrm{i}}$ states, noted $<\mathrm{C}_{\mathrm{i}}>$.

To test more systematically the validity of the estimator based on Fourier series introduced in (Eq.(8)), e.g. $C_{0}$, the periodic solutions were also computed when varying the forcing variables independently, using in some case extreme and unrealistic values. The precision of $C_{0}$ was assessed using the $\sigma / C_{0}$ ratio, where $\sigma$ is the standard deviation of the $C_{\mathrm{i}}$ states and represents the size of the oscillations characterizing the periodic solution. The bias of $C_{0}$ was assessed using the $\operatorname{abs}\left(<C_{i}>-C_{0}\right) / C_{0}$ ratio. We modeled the distributions of the forcing variables using Gaussian functions as

$$
y_{i}=y_{\min }+\frac{N_{i}(d)}{\sum_{i} N_{i}(d)} a \quad \text { with } \quad N_{i}(d)=\frac{1}{d \sqrt{2 \pi}} e^{-\frac{(i-6)^{2}}{2 d^{2}}}
$$

213 Where $y_{i}, \mathrm{i}$ in [1..12] is the value of the forcing variable (either $b(t)$ or $\rho(t)$ ) at month $i, a$ and $d$ 214 respectively the amplitude and dispersion characterizing the distributions, and $y_{\min }$ a minimum 215 value for the considered variable. Low values of the $d$ parameter yielded distributions having 216 a spike around the sixth month, high values uniform distributions. The a parameter 217 represented a scaling parameter. $a$ and $d$ were varied at once and sampled linearly within their 218 range of variation. $y_{\min }$ and the range of variation of $d$ and $a$ were, respectively for $b(t)$ and 
$\rho(t),(0.1,[0.1,5]$ and $[0.1,10])$ and $(0.83,[0.1,5]$ and $[0.1,100])$. One forcing variable

220 remained constant whilst the amplitude and dispersion of the other was varied and set to 0.2 for $b_{i}, \mathrm{i}$ in [1..12] and set to $0.8,0.8,0.8,0.82,1.06,1.10,1.06,0.82,0.8,0.8,0.8,0.8$ for $\rho_{i}, \mathrm{i}$ in $[1 . .12]$.

223 To compute the solutions with both approaches, the calculation of modifiers of the 224 decomposition rates was done using SQL requests under the PostgreSQL DBMS and further 225 calculations using the R Software. All the computations were done on a bi-xeon, 2Go RAM.

\section{Results}

227 Computation times on the standard climatic dataset were 57.9", and 46.9" for respectively the $228 C i$ (disctrete formulation) and $C_{0}$ (continuous formulation) calculations (to be compared to 229 15'10" needed when running the Fortran implementation of RothC available online at the 230 Rothamsted Research website; this time includes for each point of the climatic dataset reading the data files, running the model using the equilibrium mode and writing the results).

On the long term, temporal variability of the forcing variables resulted in oscillations of the total SOC which size reached for some points of the standard climatic dataset $11 \%$ of the total SOC, as estimated using the discrete formulation. However, over this standard climatic spectrum, all methods gave similar results regarding the average SOC value at equilibrium (maximum absolute error of 1\%). The long term values for the different carbon compartments at equilibrium were all similar but the value for the DPM compartment (Figure 1). For this compartment, the effect of the temporal variability of the forcing variable (obtained by comparing $<C_{i}>$ to the other solutions) was important. The error caused by using RothC as the discretization of the continuous formulation (see $\S 1.4$ ) can be seen when comparing $C_{a v g}$ with $C_{0}$. It appears that has $C_{a v g}$ slightly overestimates the DPM pool compared to $C_{0}$.

$C_{0}$ became strongly biased and imprecise for extreme distributions of $\rho(t)$ where amplitude of this forcing variable was high and variability over the period was large (for $a=100$ and $c=0.1$ yielding maximum $\rho$ values of 100.1, bias reached 0.5 and imprecision 0.27 , Figure 2, top diagrams). The imprecision and bias of $C_{0}$ also depended on the amplitude and dispersion of $b(t)$ but always remained small (Figure 2, bottom diagrams). We checked (not displayed here) that the bias and imprecision of $C_{0}$ compared to $C_{i}$ was not caused by using RothC as a discretization of the continuous model but by the fact that $C_{0}$ leverages the time-variability of the forcing variables when $C_{i}$ does not. Thus, the domains where $C_{0}$ is imprecise and more 
importantly biased are the domains where the time-variability of the forcing variables greatly 251 determines the behavior of the system.

\section{Conclusion}

253 The continuous formulation using Fourier transforms makes it possible to specify analytically 254 the forcing variables as functions of time, and then to obtain analytical solutions for the 255 mathematical formulation of the model of carbon dynamics under study. Here, we used a zero 256 order transform, which makes the forcing variables constant through time, and studied the 257 validity of such an assumption.

258 We showed in turn that such an approximation resulted in short computation times and is 259 reasonably precise (i) for the common application domain of the RothC model and (ii) in case 260 there is no concern about intra-annual variations of decomposable plant material and to a 261 smaller extent of the microbial community. If these conditions are not met, one may want to 262 use the discrete formulation or higher order Fourier transforms in order to grasp more of the 263 temporal variability of the variables. It is likely that in standard conditions, the use of average 264 agro-climatic conditions for computing steady state solutions of linear models of organic 265 matter dynamics, which is commonly found in literature on the subject (Bolker et al., 1998; 266 Baisden et al., 2003; Manzoni et al., 2004), can be legitimated. The Fourier series approach is 267 not restricted to linear models or to models taking only $\mathrm{C}$ dynamics into account. It could be 268 particularly relevant for non-linear systems, where assuming that forcing variable can be 269 averaged could become even more tedious than for linear systems. We also emphasize here 270 the fact that the method proposed to deal with the continuous formulation, since it essentially 271 relies on the use of Fourier series, is most suited to the modeling of periodic functions and to 272 the case where decomposition and input functions can themselves be considered as periodical.

273 The approach concerning discrete-time models was used here as a way to quickly compute the 274 long-term behavior of the discrete-time model RothC, without making any approximations 275 and thus having a larger application domain than the approach using the continuous 276 formulation. It is not likely to result in a simple analytical formulation of the equilibrium of 277 the system, because of the relative complexity of the matrix to be inversed in order to 278 compute the solution. Nevertheless, it speeds up considerably the computation compared to 279 the use of the RothC's implementation. It can be applied to more complex systems and makes 280 it possible to take into account the full time-variability of the discrete forcing variables. There 281 might be constraints on the applicability of the discrete method, aimed at ensuring the 282 solvability of the system described in Eq.(3). Determining these constraints is out of the scope 
283 of this paper but results about the Toeplitz matrices might help in this perspective. Indeed, the 284 algebraic structure of system (2) is of circulant form (or Toeplitz) and this allows the use of 285 very efficient methods for solving the eigenvalues problem we are interested in (Gray, 2006).

286 The methods proposed here to compute equilibrium solutions gave, within the RothC 287 application domain, similar results for long term SOC dynamics compared with the Fortran 288 implementation of the RothC model, while being up to 19.4 times faster. This might be 289 critical when applying the model on very large data sets, for instance those produced by 290 combining climatic, landuse and soil characteristics layers within a GIS, in order to spatially 291 compute long term SOC stocks. In addition, working at equilibrium simplifies the analysis of 292 the results as only the long-term solution is considered. Both methods are of course not 293 restricted to one-year periods and could be applied to cycles with much longer periods, for 294 instance, to crop rotations or to large climatic oscillations (with periods). It would be also 295 interesting to consider the case where, while remaining oscillatory, the forcing variables 296 exhibit a drift. This could be applied, for instance, to study the effect of climate change on 297 SOC stocks.

\section{Acknowledgments}

299 The authors thank David Coleman, Rothamsted Research, UK, for giving useful details about 300 the RothC implementation and the referees for their helpful comments. 

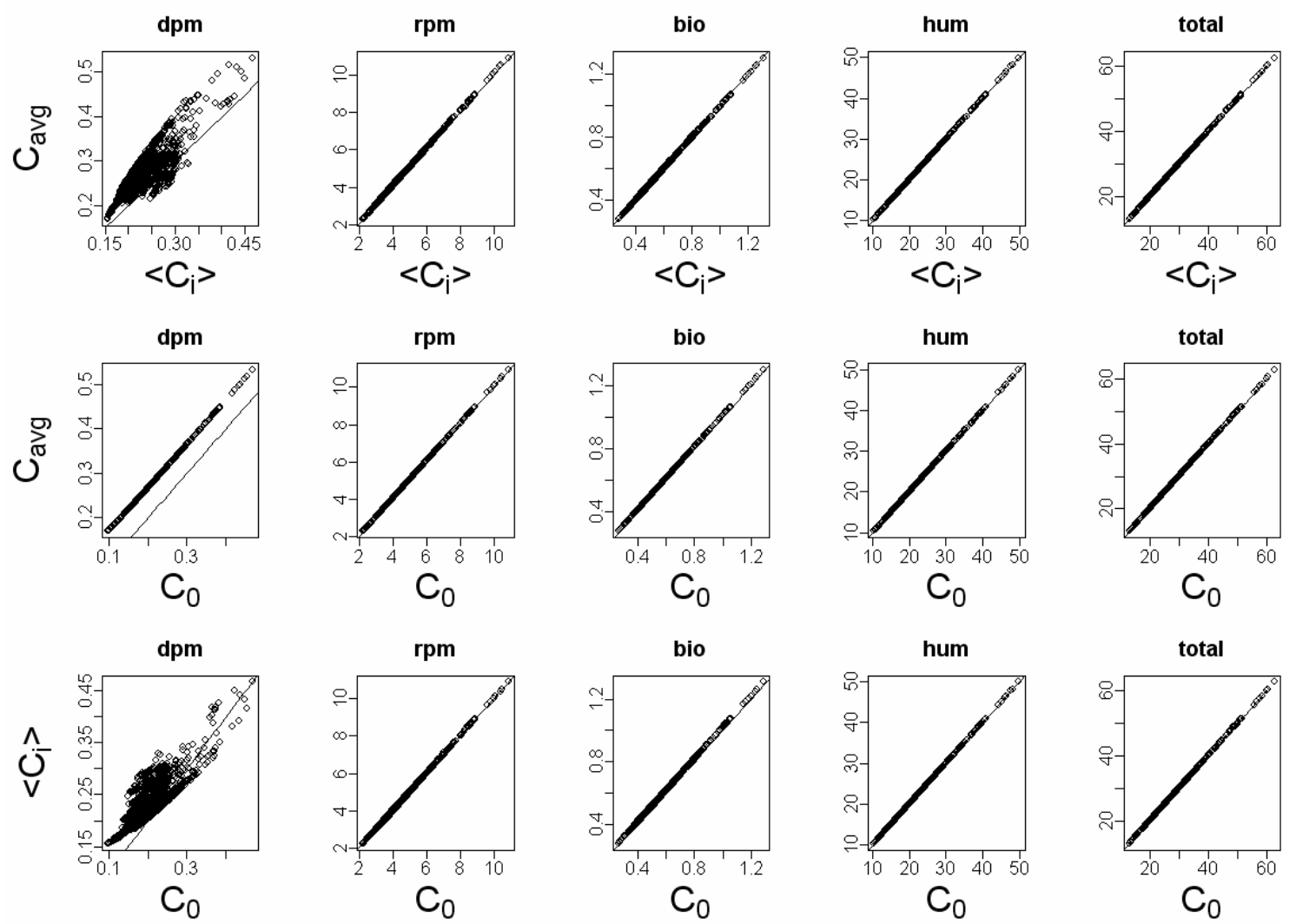

$\mathrm{C}_{0}$

$\mathrm{C}_{0}$

$\mathrm{C}_{0}$

0

302 Figure 1 : long-term solutions for each compartment of the model and for the total carbon content, over the 303 whole set of climatic conditions and with $\%$ clay $=10 \%$. All values are given in t.ha ${ }^{-1}$. First line of diagrams plots 304 the results of the discrete formulation of $\mathrm{RothC}\left(<C_{i}>\right)$ against the results obtained with the discrete formulation 305 with constant forcing variables $\left(\mathrm{C}_{\mathrm{avg}}\right)$. Line Two gives the results of the continuous form $\left(\mathrm{C}_{0}\right)$ against $\mathrm{C}_{\mathrm{avg}}$ and 306 line three $<C_{i}>$ against $\mathrm{C}_{0}$. The plain lines drawn on the charts represent the $\mathrm{y}=\mathrm{x}$ function. 

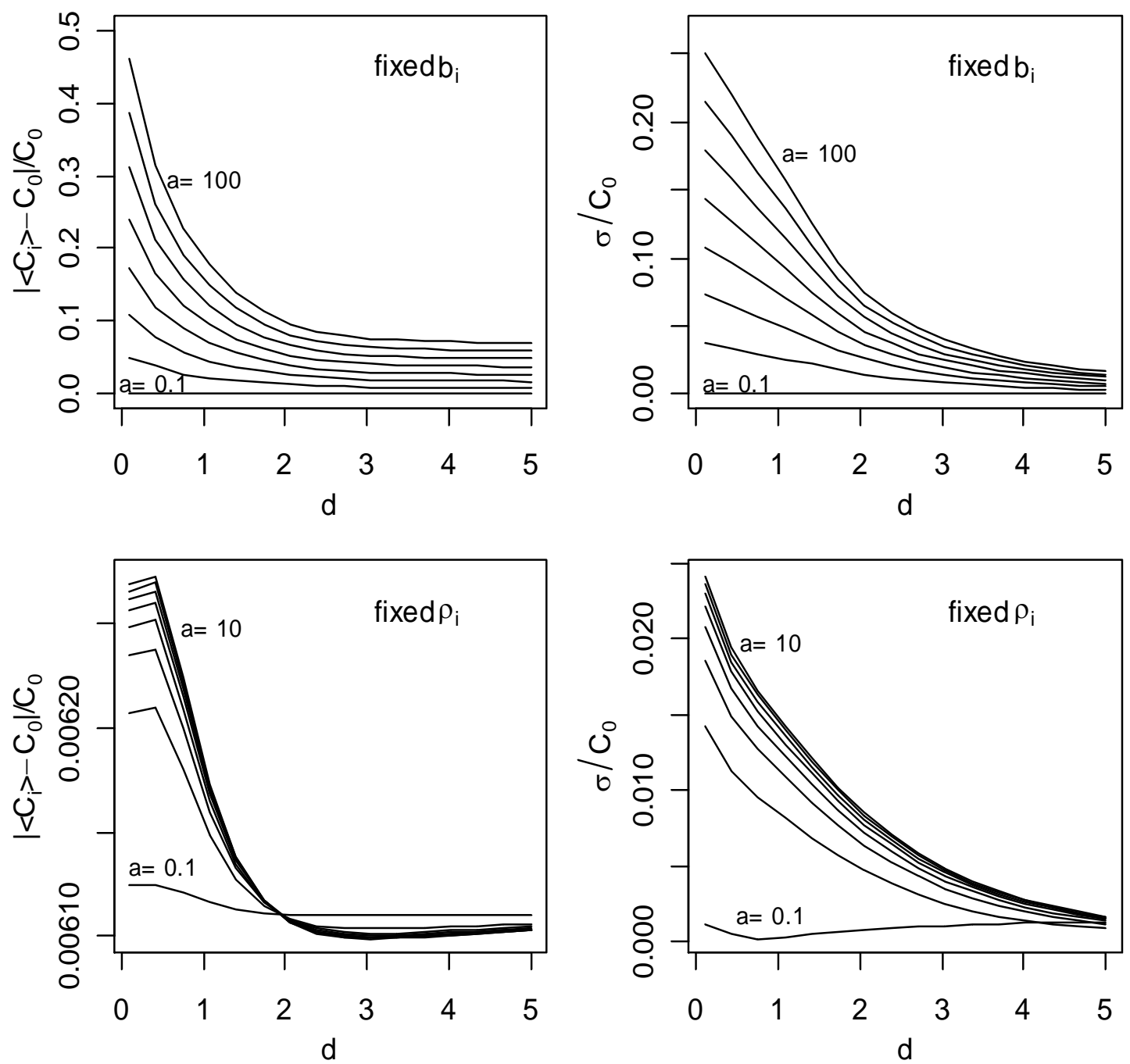

308 Figure $2:$ Left hand diagrams give the precision and right hand diagrams bias of the $C_{0}$ estimator. Top diagrams 309 are obtained keeping the sequence of monthly inputs constant and varying dispersion and amplitude of the $\rho_{i}$

310 sequence. Bottom diagrams are obtained keeping the $\rho_{i}$ sequence constant and varying dispersion and amplitude 311 of inputs over the period. $a$ and $d$ are the parameters used in Eq.(11). 


\section{References}

314

315

316

317

318

319

Baisden, W.T. and Amundson, R., 2003. An analytical approach to ecosystem biogeochemistry modeling. Ecological Applications, 13: 649-663.

Bellamy, P.H., Loveland, P.J., Bradley, R.I., Lark, R.M. and Kirk, G.J.D., 2005. Carbon losses from all soils across England and Wales 1978-2003. Nature, 437: 245-248.

Betts, R.A., 2000. Offset of the potential carbon sink from boreal forestation by decreases in surface albedo. Nature, 408: 187-190.

Bolinder, M.A., Angers, D.A. and Dubuc, J.P., 1997. Estimating shoot to root ratios and annual carbon inputs in soils for cereal crops. Agriculture Ecosystems \& Environment, 63: 61-66.

Bolker, B.M., Pacala, S.W. and Parton, W.J., 1998. Linear analysis of soil decomposition: Insights from the century model. Ecological Applications, 8: 425-439.

Bosatta, E. and Agren, G.I., 2003. Exact solutions to the continuous-quality equation for soil organic matter turnover. Journal of Theoretical Biology, 224: 97-105.

Cao, M.K. and Woodward, F.I., 1998. Dynamic responses of terrestrial ecosystem carbon cycling to global climate change. Nature, 393: 249-252.

Coleman, K. and Jenkinson, D.S., 1995. ROTHC-26.3, a model for the turnover of carbon in soil. Model description and users guide. Lawes Agricultural Trust, Harpenden.

Coleman, K., Jenkinson, D.S., Crocker, G.J., Grace, P.R., Klir, J., Korschens, M., Poulton, P.R. and Richter, D.D., 1997. Simulating trends in soil organic carbon in long-term experiments using RothC-26.3. Geoderma, 81: 29-44.

Cox, P.M., Betts, R.A., Jones, C.D., Spall, S.A. and Totterdell, I.J., 2000. Acceleration of global warming due to carbon-cycle feedbacks in a coupled climate model. Nature, 408: 184-187.

Falloon, P. and Smith, P., 2002. Simulating SOC changes in long-term experiments with RothC and CENTURY: model evaluation for a regional scale application. Soil Use and Management, 18: 101-111.

Franko, U., Schramm, G., Rodionova, V., Korschens, M., Smith, P., Coleman, K., Romanenkov, V. and Shevtsova, L., 2002. EuroSOMNET - a database for long-term experiments on soil organic matter in Europe. Computers and Electronics in Agriculture, 33: 233-239.

Gray, R.M., 2006. Toeplitz and Circulant Matrices: A review. Foundations and Trends in Communications and Information Theory, 2: 155-239.

Jenkinson, D.S., Adams, D.E. and Wild, A., 1991. Model estimates of CO2 emissions from soil in response to global warming. Nature, 351: 304-306.

Jones, C., McConnell, C., Coleman, K., Cox, P., Falloon, P., Jenkinson, D. and Powlson, D., 2005. Global climate change and soil carbon stocks; predictions from two contrasting models for the turnover of organic carbon in soil. Global Change Biology, 11: 154166.

Katterer, T. and Andren, O., 2001. The ICBM family of analytically solved models of soil carbon, nitrogen and microbial biomass dynamics descriptions and application examples. Ecological Modelling, 136: 191-207.

Knorr, W., Prentice, I.C., House, J.I. and Holland, E.A., 2005. Long-term sensitivity of soil carbon turnover to global warming. Nature, 433: 298-301.

Manzoni, S., Porporato, A., D'Odorico, P., Laio, F. and Rodriguez-Iturbe, I., 2004. Soil nutrient cycles as a nonlinear dynamical system. Nonlinear Processes in Geophysics, 11: 589-598. 
Parshotam, A., 1996. The Rothamsted soil-carbon turnover model - Discrete to continuous form. Ecological Modelling, 86: 283-289.

Shevtsova, L., Romanenkov, V., Sirotenko, O., Smith, P., Smith, J.U., Leech, P., Kanzyvaa, S. and Rodionova, V., 2003. Effect of natural and agricultural factors on long-term soil organic matter dynamics in arable soddy-podzolic soils - modeling and observation. Geoderma, 116: 165-189.

Shirato, Y., 2005. Testing the suitability of the DNDC model for simulating long-term soil organic carbon dynamics in Japanese paddy soils. Soil Science and Plant Nutrition, 51: 183-192.

Shirato, Y. and Yokozawa, M., 2005a. Applying the Rothamsted Carbon Model for long-term experiments on Japanese paddy soils and modifying it by simple mining of the decomposition rate. Soil Science and Plant Nutrition, 51: 405-415.

Shirato, Y., Paisancharoen, K., Sangtong, P., Nakviro, C., Yokozawa, M. and Matsumoto, N., 2005b. Testing the Rothamsted Carbon Model against data from long-term experiments on upland soils in Thailand. European Journal of Soil Science, 56: 179188.

Swinnen, J., Vanveen, J.A. and Merckx, R., 1995. Carbon Fluxes In The Rhizosphere Of Winter-Wheat And Spring Barley With Conventional Vs Integrated Farming. Soil Biology \& Biochemistry, 27: 811-820.

Thornton, P.E. and Rosenbloom, N.A., 2005. Ecosystem model spin-up: Estimating steady state conditions in a coupled terrestrial carbon and nitrogen cycle model. Ecological Modelling, 189: 25-48.

Vleeshouwers, L.M. and Verhagen, A., 2002. Carbon emission and sequestration by agricultural land use: a model study for Europe. Global Change Biology, 8: 519-530.

Yang, X., Wang, M.X., Huang, Y. and Wang, Y.S., 2002. A one-compartment model to study soil carbon decomposition rate at equilibrium situation. Ecological Modelling, 151: 63-73. 